\title{
Renewed Approach of Integration WLAN \& UMTS and Handover
}

\author{
Mukhwinder Kaur \\ ECE Dept. Haryana College of \\ Tech. \& Management, Kaithal, \\ India
}

\author{
Bhawna \\ ECE Dept. Haryana College of \\ Tech. \& Management, Kaithal, \\ India
}

\author{
G.C. Lall \\ ECE Dept. Haryana College of \\ Tech. \& Management, Kaithal, \\ India
}

\begin{abstract}
People all over the world want to stay connected by audio as well as video services.3G provide this facility. WLAN provides better data speeds at lower rates. In a wireless network various multiple access technologies such as wireless LAN and UMTS are used because cellular networks like UMTS provide various voice and data services and WLAN provides high speed. Integration of these two allows operators to deploy low cost and high speed WLAN to cover hotspot. Furthermore the architecture of WLAN and UMTS integration allows mobile nodes to continue data connection (ps) through WLAN and voice connection (cs) in parallel. In this paper WLAN and UMTS architecture along with its advantages and handover scheme is being proposed.
\end{abstract}

\section{Keywords}

UMTS, WLAN.IP, MN, RNC, UTRAN

\section{BASIC INTRODUCTION}

In today's modern era integrating multiple technologies is becoming essential to have desirable services available at all times. A lot of integrated technologies exist these days. Alliance of many radio based technologies not only provides good service quality but also is cost effective. That is why new generation technologies are likely to have various heterogeneous topologies to significantly improve the performance results both for network and users. Ieee $802.11 \mathrm{~b}$ is employed in agreement relieved to scientific, medical and industrial frequency band i.e. $2.4 \mathrm{GHZ}$ and it offers data rate of $11 \mathrm{mb} / \mathrm{s}$, [1] in comparison to $802.11 \mathrm{a}$ and $802.11 \mathrm{~g}$ that provides data rate equal to $54 \mathrm{mb} / \mathrm{s}$ exempting $2.4 \mathrm{GHZ}$ and $5 \mathrm{GHZ}$ bands correspondingly [2].

Ieee802.11 that is wireless local area network (wlan) has been currently implemented broadly in different areas of research. Various different technologies such as personal digital assistants (PDA's) and mobile phones are armed with wlan interface that allows users to have broadband mobile internet assistance on the go. Integrating technologies mean can connect or interface at the same time in order to have access

of more than one technology. One example of this integration is interfacing of WLAN and UMTS.WLAN is capable of providing extreme data rates at low prices. In spite of these capabilities its services are restricted to very less geographical areas. UMTS coverage artwork is huge and extensive but it is expensive and data provided by UMTS does not accomplish the constraint of high bandwidth demanding applications. In such case mechanism of integration is these two technologies will improve user applications.

\subsection{IEEE 802.11 WLAN}

Please use The IEEE standard 802.11 is usually carried out in the infrastructure and ad-hoc modes. During the infrastructure mode, the transmission between the nodes is synchronized by an Access Point (AP) within a particular radio coverage area known as service set. Infrastructure mode is related to its integration with the cellular network. One access point can be coupled with one mobile node (MN) in a given amount of time.

The communication between all of the AP's connected to MN's is handled by directly coordinating through an AP. Roaming around APs is done in layer-2 via an Inter-AP Protocol (IAPP).[9] Beacons contains cell-id called as the AP's MAC address and network-id called as Extended Service Set Identifier (ESSID) are periodically generated by the AP's along with other information. When WLAN is switched on, the MN gets connected to the AP by transmitting an associate request frame to an AP. When the MN shifts near a new cell it gets a beacon with the new cell-id but with same network-id, it then gets connected to the new AP by transmitting the re-associate request frame including an old ap's MAC address. The old AP communicates with the new AP with the assistance of IAPP [3] for downloading the framework.

Mainly two MAC functions are demarcated for 802.11 which are point co-ordinate function (pcf) and distributed coordinate function (dcf). In dcf all of the sending nodes use CSMA/CA protocol [4] to strive for the radio channel. Whenever collisions occur then frames are retransmitted by the node which is identified by the lack of acknowledgement of the receiver.

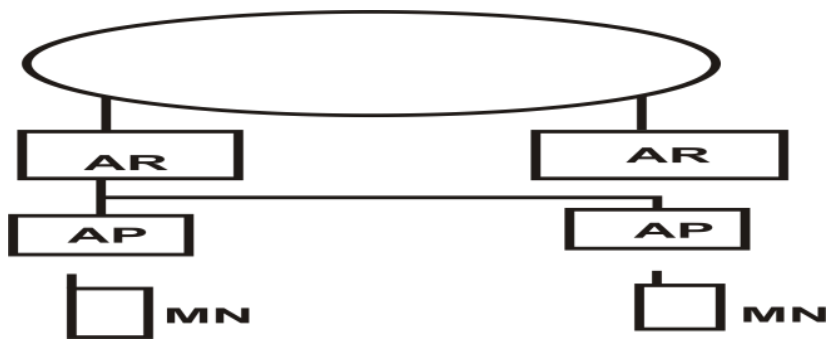

Fig.1 Wlan IP network 
In pcf the Collision Free Period (CFP) is declared by an AP when all the MNs are back off. The AP polls promises MNs in the CFP, which in turn transfer the frames. The PCF is defined for an isochronous traffic. In spite of this the PCF is not generally used for commercial APs.

\subsection{Types of Integration}

Fundamentally integration is done in three different mechanisms. Loose coupling, Tight coupling, Open coupling are the three different mechanisms of integrating the technologies.

1) Loop coupling: In this WLAN is connected to an internet back bone with the help of gateway router, hence it maintains an indirect connection. Jointly these networks are indecently operated. A very little alteration is needed for the maintenance purpose.

2) Open coupling: In open coupling both the wireless networks are not integrated in real. For example in case of WLAN and UMTS both function independently and it uses distinct authentication mechanism. It is basically employed for billing scheme.

3) Tight coupling: In this mechanism WLAN is directly connected either by ggsn or by sgsn to the umts network. Major advantages of this coupling are mobility management and to reduce handoff. But it is more difficult than loose coupling.

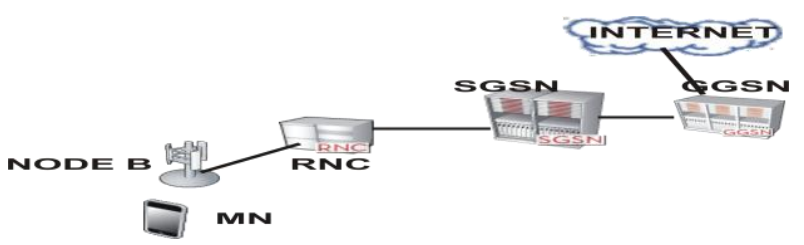

Fig.2 Integration of coupling schemes

\subsection{WLAN Protocol}

Wlan basically installs assertion centered medium access protocol (Mac) called as carrier sense multiple access protocol accomplished with collision averting (csma/cs) which is also called as energy consuming protocol. Major problem is that WLAN interfacing consumes lots of energy. Hence by reducing consumption of energy wlan interfacing can be easily done.

\subsection{Energy consumption in MAC WLAN}

Ieee802.11 standard specifies that Mac WLAN operates either in power saving mode or in active mode. In the active mode, a station is required to rouse to listen to the WLAN channel and also to transmit and receive packets. There is an access point (AP) that broadcasts the contention free protocol (CFP). The contention free mechanism is basically known as point coordination function (PCF). But when the stations do not transmit and receive then it is called a doze state.

\section{ARCHITECTURE OF UMTS}

UMTS network is mainly divided in two parts i.e. UMTS terrestrial radio access network (UTRAN) and Core network (CN).Chas two domains i.e. packet switch (PS) domain and circuit switch (CS).The diagram shows that PS-domain has gateway GPRS support node (GGSN) along with HLR and serving GPRS support node (SGSN). But UTRAN has radio network controller (RNC) performing, mobility management, encryption and radio resource management. It also manages node-B providing wireless access to mobile user.

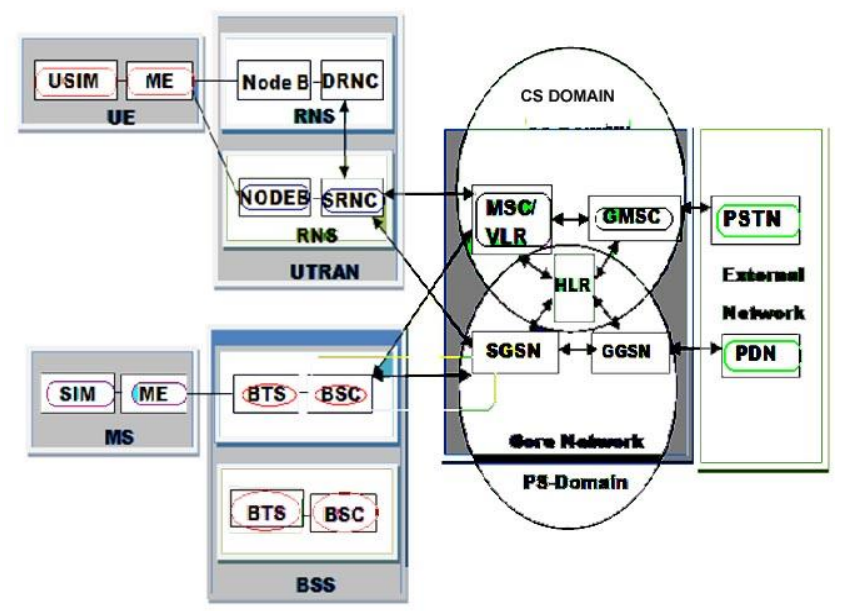

Fig.3 Architecture of UMTS

\section{ARCHITECTURE OF WLAN}

The architecture of integration of wlan 802.11 and UMTS allows a mobile node to bear voice connection across UMTS and data connection across WLAN equivalently. This becomes possible only because WLAN is mainly known for its high speed and is used for high speed data services.

WLAN is predominantly known for the asymmetric bandwidth usage in which downloading bandwidth is mainly two or three magnitude greater for e.g. in internet cafe, apartment buildings and office buildings. The cluster of high speed is known as usage area primarily recognized as hot spot. In WLAN and UMTS there is more than one point of integration. 


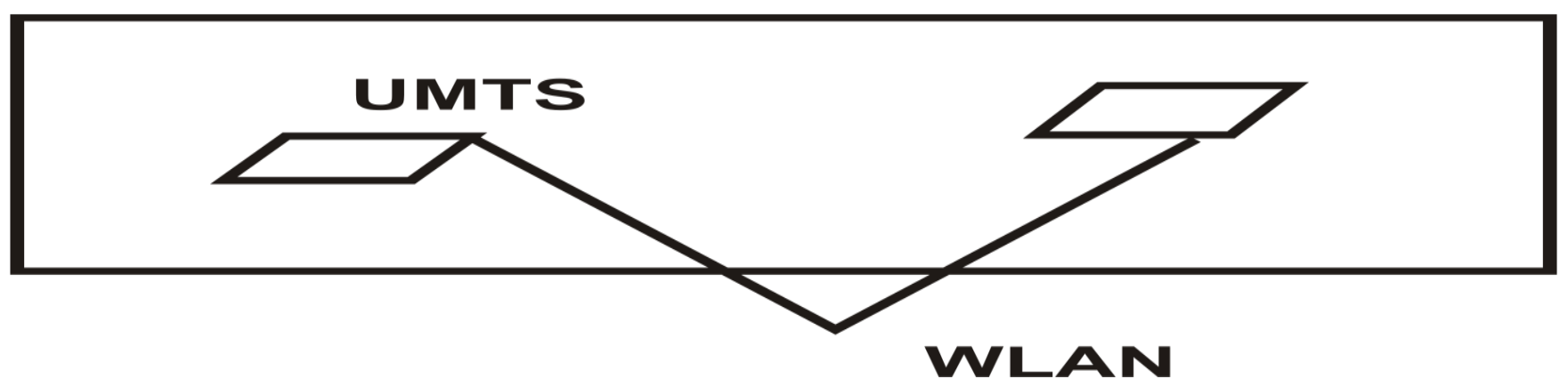

Fig.4 maximum range (Hot Spot)

\section{INTEGRATION ARCHITECTURE OF IEEE 802.11AND UMTS}

The fundamental objective behind the architecture of integration is to support the hot spot scenario. It says that whenever a MN moves towards the 802.11 micro cells, the PS connection (connection for the packet data service) by the means of UMTS RNS (or effectually with the help of GPRS) is disassembled and it is restored by the means of WLAN network. The MN uses UMTS RNS to build up the CS connection which is connection for voice service within the 802.11 cells.

Therefore it is assumed that $\mathrm{MN}$ is basically a dual-mode terminal having two different interfaces - one is 802.11 interfaces and other one is UMTS interface. Both these interfaces can be activated uniformly. The projected architecture is shown in figure 5 in which SGSN signifies the point of integration. UTRAN and WLAN are mainly two different types of radio access networks for the SGSN. Other two possibilities are evaluated before describing the integration at the SGSN.

The RNC carries out radio specific tasks such as - it converts radio frames into packets and vice versa, regulates handover and controls the radio resources. Complex radio procedures are implemented at RNC at the moment of establishing the WLAN connection because both the radio interfaces are completely different.

On the other hand, WLAN can also be attached at GGSN which makes the handover simpler from UMTS to WLAN because the GGSN simply sustains session contexts for the PS connections. On the other hand during handover to UMTS, SGSN reconstructs the mobility state while having handover with UMTS and re-establishes the pdp session and RAB framework. This is the information which is not possessed by the GGSN thereby making handover becomes slow.

The architecture of integration is displayed in Figure 5 revealing that WLAN network is connected by the means of border routers (BRs) to the SGSN. Specific signaling between the $\mathrm{MN}$ and the network is needed for having a connection through umts network for establishing and managing bearer path. WLAN network and UMTS connectivity through
Various interfaces are maintained by the $\mathrm{MN}$ in 802.11. UMTS connection is mainly depleted for the voice services. In addition UMTS connection is use for the PS signaling primarily for establishing and managing the PS connection. Therefore, the PDP context which is the surviving gprs signaling protocol is used to establish the bearer data path with the help of WLAN network.

Besides it also acquires major changes in majority of the GPRS procedures realized at the SGSN in order to differentiate both of the cases comprising of the PS bearer paths that are establish either through wlan or umts rns. In other methodology, used for the same architecture is for both of the signaling or bearer paths for the PS connections.

They are created by the means of WLAN to SGSN. The complete connection acquires little changes in few of the prevailing GPRS procedures. Independent signaling is possessed by the voice connection and bearer path without any effect on data connection. Upcoming mobile networks are developed using packet-switched architecture having a number of access technologies.

This architecture enables 3G mobile networks to get extend with different wireless access technologies based on IP like Worldwide interoperability for Microwave Access called WiMAX or Wireless Local Area Network (WLAN). Many wireless networks have different designs. Even after this, the wide range of characteristics counterpart each other making integration process very attractive. On one side there is UMTS for 3G cellular networks imparting high mobility, wide coverage area with narrow bandwidth and on other side there are many technologies for instance wlan providing less area of coverage with broad bandwidth. When both these technologies are combined they result in persistent wireless network having hotspots providing services at high speed.

Different methods have been studied [11], [12], [13] illustrating about way to combine umts and wlan and explain how handover is done among the technologies. Almost all the approaches are based on Mobile IP. Different simulations depict [11] that performance of handover with IP [12] is quite low specifically when handover is performed with some particular technology. This kind of poor performance is 


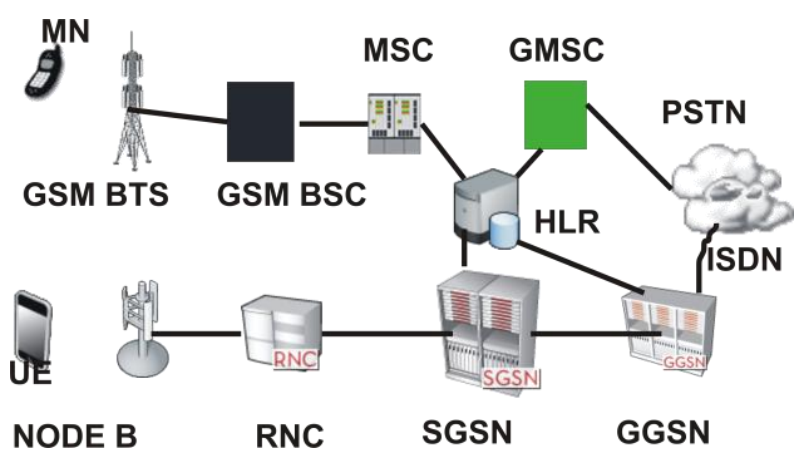

Fig.5 Integration of umts \& wlan

Mainly observed when handover is performed on the network layer. Messages are sent periodically by mobile IP for changing the connection.

Simultaneously if three messages are mislaid then only handover is done. This leads to handover delays for few seconds. A handover protocol having tight coupled architecture is used for reducing the handover delays allowing the integration of the wlan access points into the architecture of umts network.

\section{HANDOVER PROCESS FROM IEEE 802.11 TO WLAN}

In this part of paper, handover protocols from wlan to umts are explained. The network architecture is displayed in figure 6 . The integral part of umts network is wlan access points

Support node called SGSN provides a radio access network for the prevailing cellular network. [14] There are two interfaces for mobile equipment known as wlan interface and umts interface.

Both these interfaces are connected with each other and with network layer by handover module. Measurement reports signals SGSN when ME goes out of the coverage area of wlan cell and the vertical handover gets initiated.

Above diagram (Fig.6) tells about the important aspects related to vertical handover such as tunnel Management among the SGSN and the Gateway GPRS Support Node called GGSN and intercommunication. All the activities are carried out in simulation. The vertical handover consists mainly of three parts that is connection establishment, handover procedure and connection release.

To initialize the procedure an UMTS device activation request is usually transmitted from SGSN to mobile equipment via existing wlan connection. Also a Packet Data Protocol (PDP) context request is send in return by mobile equipment for quality of service class being used in wlan around the UMTS channel back to the SGSN. Finally after the completion of transmission radio Access Bearer (RAB) is installed and tunnel is created between RNC and SGSN. [15]

Then the mobile equipment gets connected with both the networks after reaching good quality of service and when activation response is transmitted to the SGSN by the UMTS network. When the connection gets established successfully the vertical handover gets initiated by SGSN.

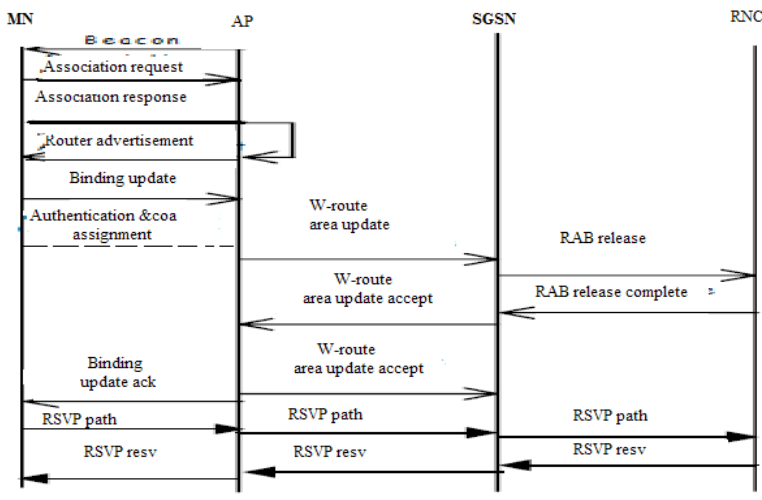

Fig.6 UMTS_WLAN handover procedure

After this ME get switched over to UMTS signaling device exchange to SGSN along with message of handover. Post that, SGSN initiates IP-over-IP tunnel to RNC. Alongside SGSN updates ME address to forward traffic to RNC. Finally wlan's old connection is de-allocated to save resources. Therefore the tunnel between the access point and SGSN is released and ME is disassociated to the access point.

\section{CHALLENGES DURING INTEGRATION OF WLAN AND UMTS}

The major challenges encountered in integrating wlan are given below

1) The impact of difference in the qos model for the two radio access network types of applications. [16], [17]

2) How to deal with variant connection paradigms that are used in every network like wlan is connection less whereas gprs is connection oriented.

3) How to certify the packet routing access.

4) How to choose the best integration point when multi network is being used.

\section{VARIOUS ADVANTAGES OF WLAN AND UMTS INTEGRATION}

1) $4 G$ networks endow many benefits to service providers and users [18] [19]. They can choose a particular network according to user's needs from available integrated networks which avoids several issues of limited capacity and no coverage.

2) $4 \mathrm{G}$ networks can be easily connected with networks having abundant resources making users connect anytime, anywhere with the network

3) Available resources of wireless networks can be utilized economically by integrating heterogeneous network each wireless network. It makes possible reusing deployed networks to provide services anytime, anywhere.

4) The WLAN-UMTS integration networks provide [20] load balancing and also avoid congestion. For instance in a network at the time of congestion, user's data is transferred to a number of integrated wireless networks. By doing this congestion can be avoided.

5) WLAN and cellular network area of coverage is increased with integration of WLAN-UMTS network. For instance, in the hotspot region UMTS user can be aided by WLAN in hotspot regions. Similarly WLAN users can also be aided by 
UMTS network when he goes away from coverage area of WLAN.

6) The integration helps in improving security features because WLAN security features provide limited security to network from various network attacks. Thus in case of integrated WLAN-UMTS network, security features of UMTS is reused for WLAN.

\section{ACKNOWLEDGMENTS}

Authors of the paper are thankful to anonymous authors and research contributor for their valuable efforts and findings.

\section{CONCLUSION}

During the course of this paper we explained briefly about integration architecture meant for umts and wlan; we also talked about wlan being used in hotspot area from microcell within umts microcells along with the UMTS architecture. We also brought forward some of the advantages and challenges of this integration and also discussed various vertical handover techniques which support and work with a lot of micro mobility solutions which are used in WLAN network. Challenges mentioned in this paper will probably help future researchers to drill down on the root causes of these challenges and also to fix these causes. Various advantages mentioned in this paper might come handy to use this technology in various spheres and expand the use of this technology.

\section{REFERENCES}

[1] W. Song, H. Jiang, W. Zhuang, and Xuemin Shen , "Resource management for Qos support in cellular/WLAN interworking," Network IEEE , vol.19 no.5, pp. 12- 18, Sept.-Oct. 2005

[2] W.Song,W.Zhuang,A.Saleh," Interworking of 3G cellular networks and Wireless LAN" ,International Journal of Wireless and Mobile Computing, vol.2, no. 4, pp. $237-247,2007$

[3] Matthew Gast, 802.11 Wireless Networks - The Definitive Guide, O’Reilly, 2002

[4] IEEE Std. 802.11b, Supplement to ANSI/IEEE Std. 802.11,1999 Edition, IEEE Standard for Wireless LAN MAC and PHY Specifications, PDF: ISBN 0-7381-18125 , January 2000

[5] Aziz, A.; Saad, N.M.; Samir, B.B.; Dept. of Elect. \& Electro Eng, Univ. Teknol. Petronas, Tronoh, Malaysia "A comparative analysis of integration schemes for UMTS and WLAN networks ",Circuits and Systems (APCCAS), 2010 IEEE Asia Pacific Conference on 6-9 Dec. 2010
[6] Christine E. Jones, Krishna M. Siva lingam, Prathima Agrawal, Jyh Cheng Chen, A survey of energy efficient network protocols for wireless networks, Wireless Networks 7 (4) (2001) 343-358

[7] A. Helmy, and M. Jaseemuddin, Efficient MicroMobility using Intra-domain Multicast-based Mechanisms (M\&M), USCCS-TR-01-747, August 2001

[8] A Comparative Analysis of Integration Schemes for UMTS and WLAN Networks Safdar Rizvi, Asif Aziz, N.M. Saad, Brahim Belhaouari Samir, Department of Electrical and Electronic Engineering, University Technology Petrona 31750 Tronoh, Perak, Malaysia, 978-1-4244-7456-1/10, 2010 IEEE

[9] M.A. Amara, "Performance of WLAN and UMTS integration at the hot spot location using opnet", 2003 2006

[10] An Architecture for Integrating UMTS and 802.11 WLAN Networks", Muhammad Jaseemuddin Dept. of Electrical \& Computer Engineering, Ryerson University, 2009

[11] J. Alba-Laurila, J. Mikkonen, and J. Rinnemaa, Wireless LANAccess Network Architecture for Mobile Operators, IEEE Communications, pp. 82-89, Vol. 39, No. 11, November 2001

[12] A.K. Salkintzis, "Interworking techniques and architectures for WLAN/3G integration toward 4G mobile data networks," Wireless Communications, IEEE, vol.11, no.3, pp. 50- 61, June 2004

[13] Vahid Solouk, Borhanuddin Mohd Ali, Daniel Wong "Vertical Fast Handoff in Integrated WLAN and UMTS Networks ", ICWMC 2011, the Seventh International Conference on Wireless and Mobile Communications 2011

[14] Qixiang Pang, S.C. Liew, V.C.M. Leung, Performance improvement of 802.11Wireless network with TCP ACK agent and auto-zoom backoff algorithm, in: IEEE Vehicular Technology Conference, 2005

[15] Zhi Ren, Guangyu Wang, Qianbin Chen, Hongbin Li" Modeling and simulation of Rayleigh fading, path loss, and shadowing fading for wireless mobile networks", Simulation Modeling Practice and Theory 19 (2011)

[16] A. H. Zahran, B. Liang, A. Saleh, "Signal Threshold Adaptation for Vertical Handoff in Heterogeneous Wireless Networks". Mobile Networks and Applications, 11: 625-640, 2006

[17] Celal Ceken, Serhan Yarkan „Huseyin Arslan,' Interference aware vertical handoff decision algorithm for quality of service support in wireless heterogeneous networks", Computer Networks 54 (2010) 